\title{
Citrate-Stabilized Gold Nanorods-Directed Osteogenic Differentiation of Multiple Cells
}

This article was published in the following Dove Press journal:

International Journal of Nanomedicine

\author{
Yibo Zhang ${ }^{1,2, *}$ \\ Yawen $\mathrm{Li}$ iD $^{3, *}$ \\ Wei Liao ${ }^{4, *}$ \\ Wenzao Peng 5 \\ Jianghui Qin $^{2}$ \\ Dongyang Chen ${ }^{2}$ \\ Liming Zheng ${ }^{2}$ \\ Wenjin Yan ${ }^{2}$ \\ Lan $\mathrm{Li}^{2}$ \\ Zhirui Guo ${ }^{3}$ \\ Peng Wang ${ }^{2,6}$ \\ Qing Jiang ${ }^{1,2}$
}

'Nanjing Drum Tower Hospital Clinical College of Nanjing Medical University, Nanjing, 210008, People's Republic of China; ${ }^{2}$ State Key Laboratory of Pharmaceutical Biotechnology, Department of Sports Medicine and Adult Reconstructive Surgery, Nanjing Drum Tower Hospital, The Affiliated Hospital of Nanjing University Medical School, Nanjing, 210008, People's Republic of China; ${ }^{3}$ Lab Center, The Second Affiliated Hospital of Nanjing Medical University, Nanjing, 210029, People's Republic of China; ${ }^{4}$ Children's Hospital of Nanjing Medical University, Nanjing, 210008, People's Republic of China; ${ }^{5}$ Jiangsu Key Laboratory of Oral Diseases, Department of Periodontics, Affiliated Hospital of Stomatology, Nanjing Medical University, Nanjing, People's Republic of China; ${ }^{6}$ State Key Laboratory of Bioelectronics, Jiangsu Key Laboratory for Biomaterials and Devices, School of Biological

Science and Medical Engineering, Southeast

University, Nanjing, 210009, People's Republic of China

*These authors contributed equally to this work

Correspondence: Zhirui Guo

The Second Affiliated Hospital, Nanjing

Medical University, Jiangsu, 210029,

People's Republic of China

Email zhiruiguo@njmu.edu.cn

Qing Jiang

State Key Laboratory of Pharmaceutical Biotechnology, Department of Sports

Medicine and Adult Reconstructive

Surgery, Nanjing Drum Tower Hospital,

The Affiliated Hospital of Nanjing

University Medical School, No. 32I

Zhongshan Road, Nanjing, 210008,

People's Republic of China

Email qingj@nju.edu.cn
Objective: Gold nanorods (AuNRs) show great potential for versatile biomedical applications, such as stem cell therapy and bone tissue engineering. However, as an indispensable shape-directing agent for the growth of AuNRs, cetyltrimethylammonium bromide (CTAB) is not optimal for biological studies because it forms a cytotoxic bilayer on the AuNR surface, which interferes with the interactions with biological cells.

Methods: Citrate-stabilized AuNRs with various aspect-ratios (Cit-NRI, Cit-NRII, and CitNRIII) were prepared by the combination of end-selective etching and poly(sodium 4-styrenesulfonate)-assisted ligand exchange method. Their effects on osteogenic differentiation of the pre-osteoblastic cell line (MC3T3-E1), rat bone marrow mesenchymal stem cells (rBMSCs), and human periodontal ligament progenitor cells (PDLPs) have been investigated. Potential signaling pathway of citrate-stabilized AuNRs-induced osteogenic effects was also investigated.

Results: The experimental results showed that citrate-stabilized AuNRs have superior biocompatibility and undergo aspect-ratio-dependent osteogenic differentiation via expression of osteogenic marker genes, alkaline phosphatase (ALP) activity and formation of mineralized nodule. Furthermore, Wnt/ $\beta$-catenin signaling pathway might provide a potential explanation for the citrate-stabilized AuNRs-mediated osteogenic differentiation. Conclusion: These findings revealed that citrate-stabilized AuNRs with great biocompatibility could regulate the osteogenic differentiation of multiple cell types through Wnt/ $\beta$ catenin signaling pathway, which promote innovative AuNRs in the field of tissue engineering and other biomedical applications.

Keywords: citrate-stabilized, gold nanorods, osteogenic differentiation, multiple cells, Wnt/ $\beta$-catenin signaling pathway

\section{Introduction}

Nanomaterials have brought innovation in the field of nanomedicine due to their superior biocompatibility, distinct physicochemical properties and easy functionalization processes. ${ }^{1-3}$ Recently, there is accumulating evidence that nanomaterials can modulate fate of cells and interactions between nanomaterials and biological cells have attracted considerable attention in facilitating stem cell therapy and bone tissue engineering. ${ }^{4-6}$ Nanomaterials can flexibly and controllably manipulate biological process of cells to differentiate toward desired lineages. Particularly, nanomaterials with various composition, size, shape or surface chemistry have been widely used as promising osteogenic agents in accelerating osteogenic differentiation of biological cells and bone tissue regeneration. ${ }^{7,8}$ Therefore, the possible applications of nanomaterials in regenerative medicine, especially bone tissue engineering, have been widely studied. 
Among the previously reported nanomaterials, colloidal single-crystalline gold nanorods (AuNRs) have gained increasing attention due to their widely tunable optical (nanoplasmonics) and electronic properties, which make them suitable for a wide range of applications, including solar harvesting, photovoltaics, surface-enhanced spectroscopies, diagnostics, imaging, and therapy. ${ }^{9-11}$ Particularly, the one-dimensional structure of anisotropic AuNRs exhibit two distinct plasmon bands: the longitudinal plasmon resonance (LSPR) and the transverse plasmon resonance (TSPR). The multimodal nature endows AuNRs displaying extensively application prospect in bioimaging or photothermal therapy. The most classical protocol for the preparation of the pristine is the seed-mediated growth method, in which cetyltrimethylammonium bromide (CTAB) is used as a surfactant to build a tightly bound bilayer. However, in terms of biological applications, the surface modification of the pristine AuNRs is essential due to the potential toxicity of CTAB around the AuNR surface. $^{12,13}$

A common solution is coating the CTAB bilayer with a biocompatible material, such as polyelectrolyte, lipid, silica, gel, and protein, to reduce the toxicity of CTAB on the original AuNR surface. ${ }^{14-16}$ Accumulating evidence suggested that such modified AuNRs facilitates bone tissue engineering. Vieira et al reported that gellan gumcoated AuNRs have a positive influence on osteogenesis. ${ }^{17}$ However, while these direct coating strategies markedly improve biocompatibility, the thick and dense surface modification coating heavily hinder their physicochemical properties and interactions with biological cells since it has evidenced that these AuNRs are enclosed by many high-index crystalline facets. ${ }^{18}$ Therefore, an alternative AuNR candidate with optimal biocompatibility as well as an accessible surface is essential. Wei et al reported a robust surfactant exchange method for converting CTAB-stabilized AuNRs into citrate-capped AuNRs, utilizing polystyrene sulfonate (PSS) as a mild detergent and citrate as a stabilizing agent. ${ }^{19}$ Among diverse surface ligands, citrate plays a critical role because it provides a thin, loosely and moderately bound layer, which can be easily be approached, despite its good biocompatibility. In addition, there is accumulating evidence that behaviors of AuNRs in biological systems can be manipulated by their sizes. Chen's group reported that bovine serum albumin (BSA)-modified $\mathrm{Au}$ NRs with sizes of 40, 70 and 110 nm showed excellent compatibility, and their effects on osteogenic differentiation of human mesenchymal stem cells (hMSCs) were further investigated, where $70 \mathrm{~nm}$ AuNRs significantly increased but blocked by $40 \mathrm{~nm}$ AuNRs. ${ }^{20}$ However, the present methods for turning aspect ratio (AR) of AuNRs were accomplished via varying the experimental parameters during the synthetic process, which may not guarantee their reliability and reproducibility. Our previous study suggested a rapid and robust anisotropic oxidation method for selectively shortening AuNRs with $\mathrm{AuBr}_{4}$-CTA complex oxidation under mild temperature. ${ }^{21}$ The citrate-stabilized Au NRs with desired and precise aspect ratio can be achieved. In this regard, it is imperative to evaluate the cellular effects of citrate-stabilized AuNRs with various sizes. Till now, to the best of our knowledge, the effects of citrate-stabilized AuNRs with various aspect-ratios on osteogenic differentiation of cells have not been investigated.

In this study, citrate-stabilized AuNRs with various aspect-ratios (Cit-NRI, Cit-NRII, and Cit-NRIII) were prepared using surfactant exchange strategy and end-selective etching method. The as-prepared citrate-stabilized AuNRs showed excellent biocompatibility compared to CTABstabilized AuNRs. Furthermore, their effects on osteogenic differentiation of MC3T3-E1 cells, rBMSCs and PDLPs were comprehensively investigated through the expression of osteogenic marker genes, alkaline phosphatase (ALP) activity and the formation of mineralized nodule. We also investigated the potential signaling pathway involved in citrate-stabilized AuNRs-mediated osteogenic differentiation of Multiple Cells (see Scheme 1).

\section{Materials and Methods Materials}

CTAB (99\%) and PSS (Mw 70,000 Da) were purchased from Sigma. Hydrogen tetrachloroauric acid $\left(\mathrm{HAuCl}_{4} \cdot 4 \mathrm{H}_{2} \mathrm{O}, 99 \%\right)$, hydrochloric acid $(\mathrm{HCl}, 37 \mathrm{wt} \%)$, silver nitrate $\left(\mathrm{AgNO}_{3}\right)$, sodium borohydride $\left(\mathrm{NaBH}_{4}, 96 \%\right)$, trisodium citrate $\left(\mathrm{C}_{6} \mathrm{H}_{5} \mathrm{Na}_{3} \mathrm{O}_{7} \cdot 2 \mathrm{H}_{2} \mathrm{O}\right)$, sodium chloride $(\mathrm{NaCl})$, and L-ascorbic acid (L-AA) were obtained from Shanghai Chemical Reagent Co., Ltd (China). The Milli-Q water with resistance $18 \mathrm{~m} \Omega$ was used in all experiments. All glassware was cleaned by aqua regia $\left(\mathrm{HCl} / \mathrm{HNO}_{3}\right.$ in $\left.3: 1 \mathrm{v} / \mathrm{v}\right)$ before usage.

\section{Synthesis of Colloidal Gold Seeds}

Freshly prepared $0.1 \mathrm{~mL} \mathrm{HAuCl}{ }_{4} \cdot 4 \mathrm{H}_{2} \mathrm{O}(0.25 \mathrm{mM})$ solution was added to $10 \mathrm{~mL}$ CTAB $(10 \mathrm{mM})$ aqueous solution. The newly configured $\mathrm{NaBH}_{4}$ was added to the above 


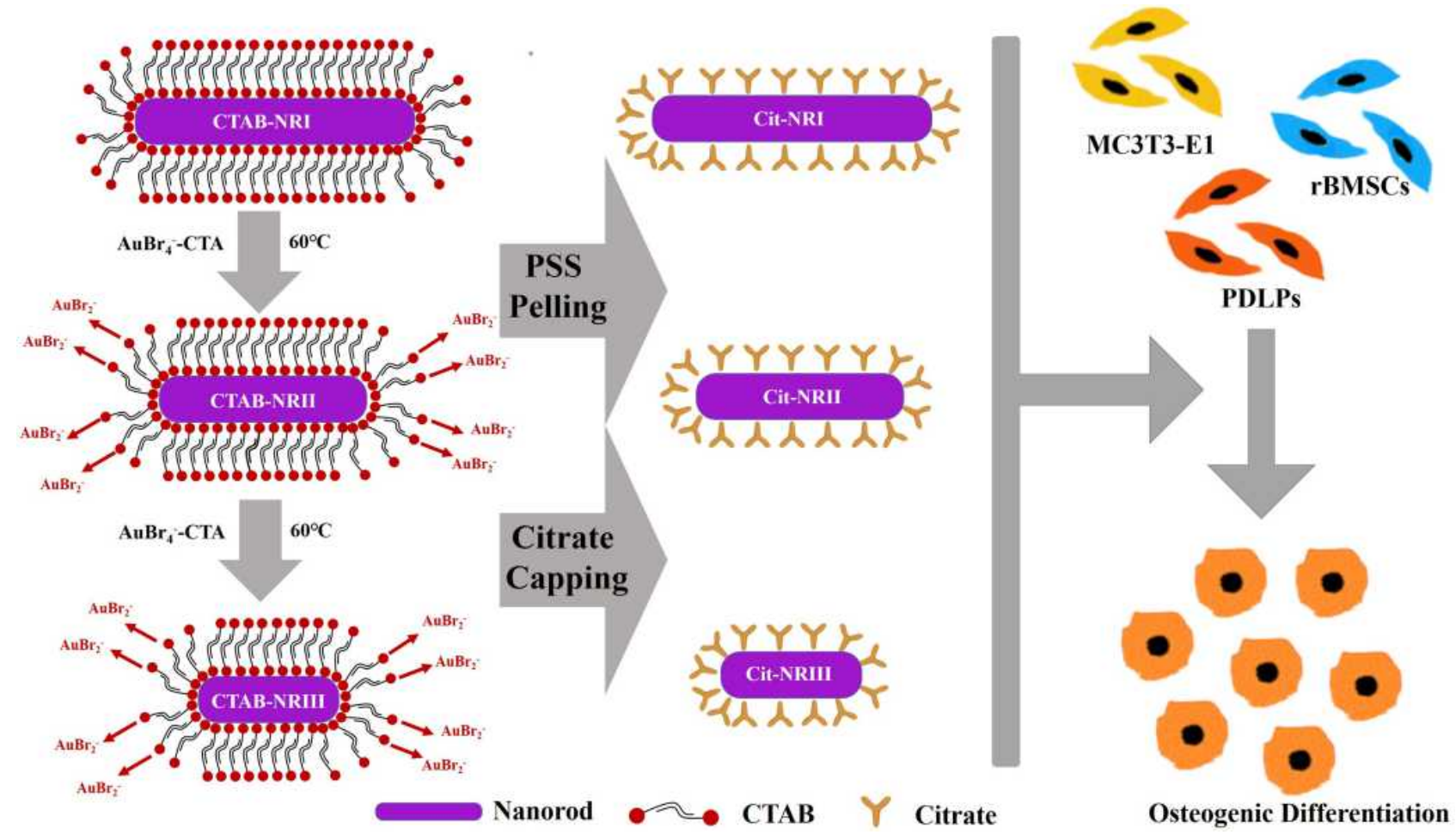

Scheme I Schematic illustration of citrate-stabilized AuNRs and their effects on osteogenic differentiation of MC3T3-EI cells, rBMSCs, and PDLPs.

mixture with agitation for $2 \mathrm{~min}$; the color of the solution turned bright yellow to brown immediately. The seed solution was stored at room temperature for at least 2 $\mathrm{h}$ to guarantee complete hydrolyzation of the unreacted $\mathrm{NaBH}_{4}$.

\section{Preparation of CTAB-Stabilized AuNRs with Different Aspect-Ratios}

CTAB-NRI was synthesized by the seed growth method. ${ }^{12,22}$ Typically, $1 \mathrm{~mL} \mathrm{AgNO}_{3}$ aqueous solution $(10 \mathrm{mM}), 2 \mathrm{~mL} \mathrm{HAuCl}_{4}$ aqueous solution (1 $\mathrm{wt} \%$ ), $0.8 \mathrm{~mL} \mathrm{HCl}$ aqueous solution $(1 \mathrm{M})$, and $0.55 \mathrm{~mL} \mathrm{L-AA}$ aqueous solution $(0.1 \mathrm{M})$ were added sequentially mixed under slow stirring. After $1 \mathrm{~min}, 180 \mu \mathrm{L}$ seeds were added and incubated overnight in a $25^{\circ} \mathrm{C}$ water bath.

CTAB-NRII and CTAB-NRIII were prepared by the oxidation-etching method from CTAB-NRI. ${ }^{21}$ Briefly, the CTAB-NRI was suspended in 0.1 M CTAB for centrifugation. The aqueous $\mathrm{HAuCl}_{4}$ solution was added to $15 \mathrm{~mL}$ GNRs solution with an optical density (OD) $=2$ at $60{ }^{\circ} \mathrm{C}$ for $15 \mathrm{~min}$, followed by centrifugation in $2 \mathrm{mM} \mathrm{CTAB}$ solution. The amount of $\mathrm{HAuCl}_{4}$ during the preparation of CTAB-NRII and CTAB-NRIII was 70 and $140 \mu \mathrm{L}$, respectively.

\section{Preparation of Citrate-Stabilized AuNRs}

The sodium citrate-coated AuNRs were prepared by the PSS replacement method. ${ }^{23}$ CTAB-stabilized AuNRs with various aspect-ratios were harvested by centrifugation to reduce the concentration of CTAB. Then, $3 \mathrm{~mL}$ PSS (10 g/ $\mathrm{L}$ in $5 \mathrm{mM} \mathrm{NaCl}$ ) was mixed with $30 \mathrm{~mL}$ AuNRs solution (optical density $(\mathrm{OD})=3$ ). A small amount of $\mathrm{NaCl}$ solution promotes the unfolding of the charged PSS chain, which was incubated at room temperature for 1 h. After centrifugation at $6000 \mathrm{~g}$ for $15 \mathrm{~min}$, the solution was finally resuspended in $30 \mathrm{~mL}$ of deionized water. This treatment was repeated, the pellet was reconstituted in $26.3 \mathrm{~mL}$ water and $3.3 \mathrm{~mL}$ sodium citrate $(1 \mathrm{wt} \%)$ incubated for $12 \mathrm{~h}$, followed by centrifugation at $5500 \times \mathrm{g}$ for $15 \mathrm{~min}$ and reconstitution with $13.35 \mathrm{~mL}$ water and $1.65 \mathrm{~mL}$ of citrate solution.

\section{Cell Culture}

MC3T3-E1 cells were purchased from the Institute of Life Science Cell Culture Center (Shanghai, China), rBMSCs were purchased from Cyagen Biosciences Inc. (CA, USA), PDLPs were purchased from the Institute of ScienCell Research Laboratories (Carlsbad, CA, USA). The cells were cultured in growth medium (GM), containing $\alpha$ MEM medium (Gibco, USA) supplemented with 1\% 
penicillin-streptomycin solution (P/S, Gibco) and 10\% fetal bovine serum (FBS, Gibco, USA) at $37{ }^{\circ} \mathrm{C}$ in $5 \%$ $\mathrm{CO}_{2}$. For osteogenic induction, cells were cultured in an medium which containing GM supplemented with $0.1 \mu \mathrm{M}$ dexamethasone, $50 \mu \mathrm{g} / \mathrm{mL}$ ascorbic acid and $10 \mathrm{mM} \beta$ glycerophosphate.

\section{Cell Viability Assay}

The cell viability was tested using the Cell Counting Kit-8 (CCK-8) assay. MC3T3-E1 cells, rBMSCs, and PDLPs were seeded in 96 -well plates at a density of $5 \times 10^{3}$ cells/ well and cultured at $37{ }^{\circ} \mathrm{C}$ with $5 \% \mathrm{CO}_{2}$ for $24 \mathrm{~h}$, followed by incubation with AuNRs of various aspect-ratio at the final concentration of 1,10 , and $100 \mathrm{nM}$, respectively. After 1-, 3-, and 5-day incubation, CCK-8 reagent (Bimake, China) was added to each well and cultured at $37{ }^{\circ} \mathrm{C}$ for $1 \mathrm{~h}$. The OD was measured at $450 \mathrm{~nm}$ on a microplate reader (Multiskan GO, Thermo Fisher Scientific, USA).

\section{ALP Activity Assay and Staining}

After osteogenic induction for 14 days, MC3T3-E1 cells, rBMSCs, and PDLPs treated with citrate-stabilized AuNRs of various aspect-ratio were harvested. The ALP activity levels were measured using Alkaline Phosphatase Assay Kit (Beyotime, China), and the absorbance was measured at $405 \mathrm{~nm}$. For ALP staining, after osteogenic induction for 14 days, the cells were stained with BCIP/ NBT Alkaline Phosphatase Color Development Kit.

\section{Alizarin Red S Staining Assay}

After osteogenic induction for 21 days, MC3T3-E1 cells, rBMSCs, and PDLPs treated with citrate-stabilized AuNRs of various aspect-ratio were treated with 5\% alizarin red S (ARS; Sigma) staining solution for $5 \mathrm{~min}$. Subsequently, the cells were washed and examined, and images captured by an inverted optical microscope (Olympus IMT-2, Tokyo, Japan), and the formation of mineralized matrix nodules was quantified by measuring the absorbance at $562 \mathrm{~nm}$ after incubation with $10 \%(\mathrm{w} / \mathrm{v})$ cetylpyridinium chloride (Sigma-Aldrich) for $2 \mathrm{~h}$.

\section{Real-Time Quantitative PCR}

MC3T3-E1 cells were cultured in 6 -well plate $\left(3 \times 10^{5}\right.$ cells/well) for $24 \mathrm{~h}$ and incubated with citrate-stabilized AuNRs of various aspect-ratio for 3, 5, and 7 days, respectively. Then, total RNA was isolated from cells using RNA-Quick Purification Kit (Yishan Biotech, Shanghai,
China), and cDNA was generated using a HiScript II Q RT SuperMix and used as a template for qPCR (Vazyme Biotech, Nanjing, China). The ChamQ ${ }^{\mathrm{TM}}$ SYBR Color qPCR Master Mix (Vazyme Biotech) was utilized for qPCR reactions. The amplification was conducted on a LightCycler 480-II (Roche, Mannheim, Germany) using the primers listed in Table S1.

\section{Western Blot (WB) Analysis}

MC3T3-E1 cells were cultured with citrate-stabilized AuNRs of various aspect-ratio for 3 days and lysed in RIPA lysis buffer supplemented with $1 \mathrm{mM}$ Phenylmethanesulfonyl fluoride (PMSF). The proteins were obtained according to the mammalian protein extraction kit, separated by $10 \%$ SDS-PAGE, and electroblotted onto Polyvinylidene Fluoride (PVDF) membranes (Millipore Co., Billerica, MA, USA). Then, the membranes were blocked with $5 \%(\mathrm{w} / \mathrm{v})$ non-fat powdered milk for $1 \mathrm{~h}$ at room temperature, probed with primary antibody at $4{ }^{\circ} \mathrm{C}$ overnight, followed by incubation with horseradish peroxidase (HRP)-labeled secondary antibody for $1 \mathrm{~h}$. The level of protein was detected using ECL plus WB detection system.

\section{Statistical Analysis}

The differences between groups were determined using one-way analysis of variance (ANOVA), followed by Tukey's posthoc analysis. $\mathrm{P}<0.05$ indicated statistical significance. All statistical analyses were performed using GraphPad Prism 8.

\section{Results and Discussion \\ Characterization of CTAB and Citrate-Stabilized AuNRs}

CTAB-stabilized AuNRs with various aspect-ratios (CTAB-NRI, CTAB-NRII, and CTAB-NRIII) were prepared using the seed growth method and end-selective etching method. ${ }^{24,25}$ The oxidation mechanism AuNRs is attributed to $\mathrm{Au}(\mathrm{III})$ in the presence of $\mathrm{CTAB}$, where ligand exchange from $\mathrm{AuCl}_{4}^{-}$to $\mathrm{AuBr}_{4}^{-}$will occur when CTAB-modified AuNRs are mixed with HAuCl4 solution (Equation 1). The formation of AuBr4-CTA complex will facilitate the comproportionation reaction between $\mathrm{Au}(\mathrm{III})$ and $\mathrm{Au}(0)$ (Equation 2), thus shortening the AuNRs. 


$$
\mathrm{AuCl}_{4}^{-} \leftrightarrow \mathrm{AuCl}_{3} \mathrm{Br}^{-} \leftrightarrow \mathrm{AuCl}_{3} \mathrm{Br}_{2}^{-} \leftrightarrow \mathrm{AuCl} \mathrm{Br}_{3}^{-} \leftrightarrow \mathrm{AuBr}_{4}^{-}
$$

$$
\begin{aligned}
& \mathrm{AuBr}_{4}-\mathrm{CTA}+2 \mathrm{Au}+2 \mathrm{CTA}^{+}+2 \mathrm{BR}^{-} \\
& \quad=3 \mathrm{AuBr}_{2}-\mathrm{CTA}
\end{aligned}
$$

Citrate-stabilized AuNRs with various aspect-ratio (CitNRI, Cit-NRII, and Cit-NRIII) were prepared by a PSSassisted ligand exchange process from CTAB-stabilized AuNRs. ${ }^{23}$ The morphology of the synthesized nanoparticles was characterized by transmission electron microscopy (TEM), which showed that both CTAB-stabilized AuNRs (Figure 1A-C) and citrate-stabilized AuNRs (Figure 1D-F) had the same width while the length decreased gradually owing to end-selective etching method. As shown in Figure $1 \mathrm{G}$, the sizes according to the TEM images were $86.68 \pm 9.24(94.62 \pm 6.63), 76.78$ \pm 6.28 (77.18 \pm 7.84$)$, and $52.45 \pm 4.15(53.77 \pm 5.04) \mathrm{nm}$ for
CTAB-NRI (Cit-NRI), CTAB-NRII (Cit-NRII), and CTAB-NRIII (Cit-NRIII), respectively. These findings indicated that the exchange process did not affect either the morphology or the size of the AuNRs. Strikingly, the LSPR spectrum of nanoparticles indicated aggregation and surface surrounding. Therefore, the LSPR spectrum was used to monitor the colloidal stability of GNRs during the change from CTAB bilayer to citrate. $^{23}$ Based on the normalized UV-Vis spectra (Figure 1H), their absorption peaks were located at $1176 \mathrm{~nm}, 973 \mathrm{~nm}$, and $784 \mathrm{~nm}$ for CTAB-NRI, CTAB-NRII, and CTAB-NRIII, respectively. The narrow width and sharp profile of the LSPR band indicated the monodispersity of the CTAB-stabilized GNRs. After the surface exchange, the UV-vis spectra of citrate-stabilized AuNRs showed an obvious blue shift of $37.3 \mathrm{~nm}$ for Cit-NRI, $27.9 \mathrm{~nm}$ for Cit-NRII, and $18.7 \mathrm{~nm}$ for Cit-NRIII. The wavelength $\left(\lambda_{\max }\right)$ of longitudinal
A

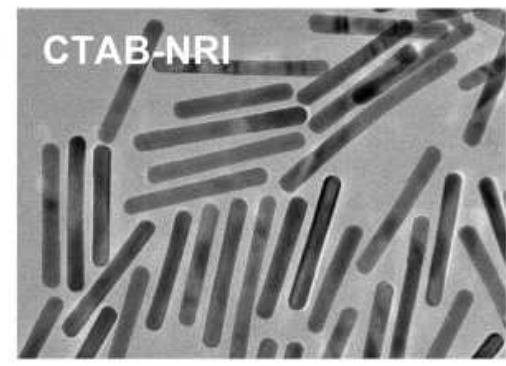

D

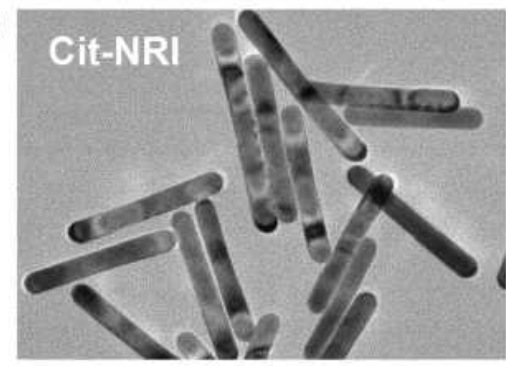

G

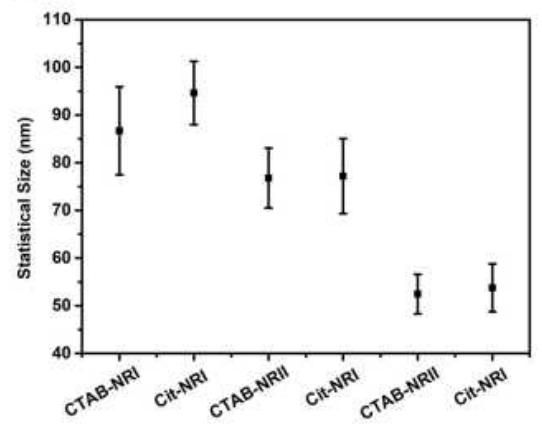

B

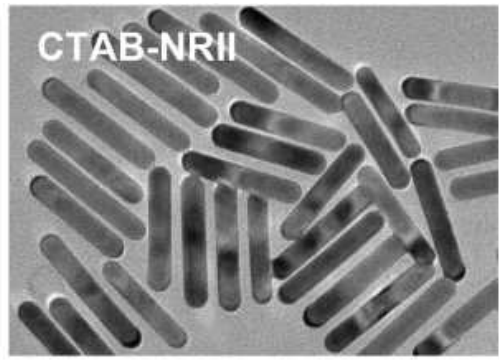

E

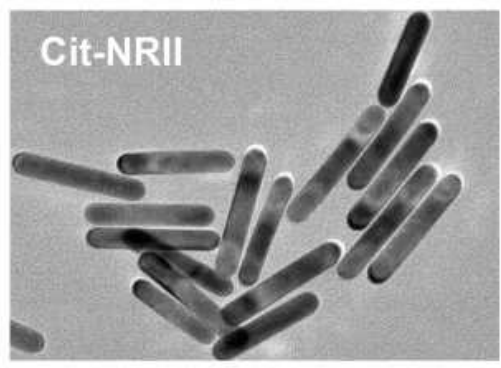

H

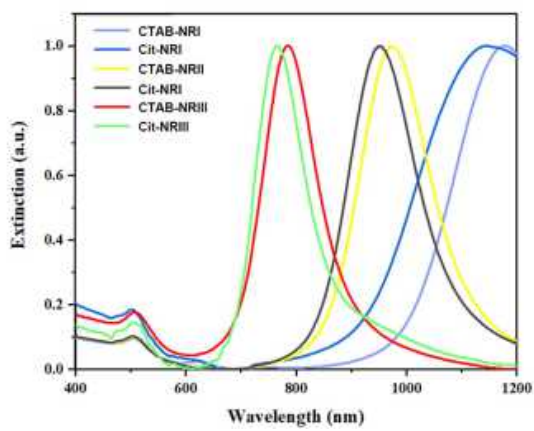

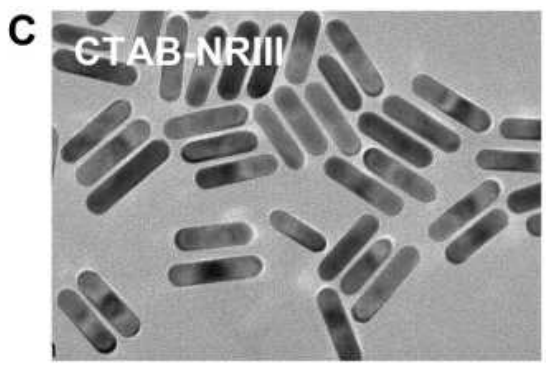

$\mathbf{F}$
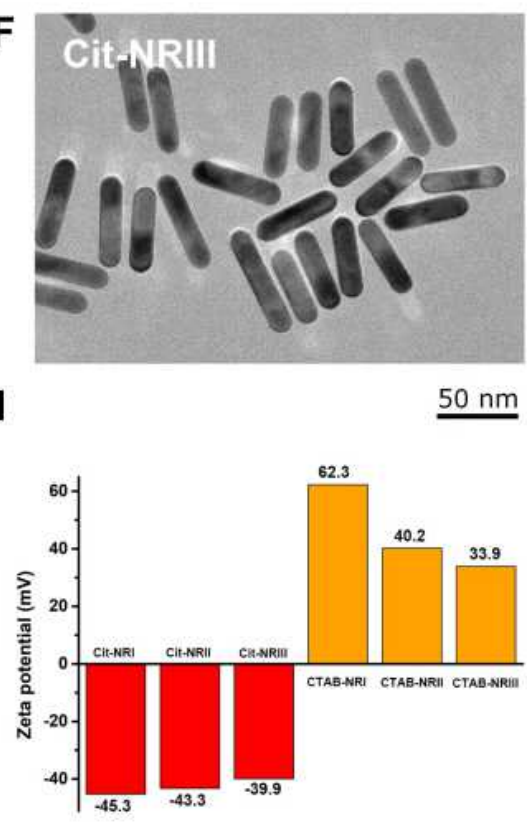

Figure I Characterization of AuNRs. Typical TEM images of as-prepared (A-C) CTAB-stabilized AuNRs and (D-F) citrate-stabilized AuNRs with various aspect-ratio. (G) Statistical Size of CTAB and citrate-stabilized AuNRs with various aspect-ratio according to the TEM images in (A-F). (H) UV-Vis absorption spectra of CTAB and citratestabilized AuNRs with various aspect-ratio. (I) Zeta potential analysis of AuNRs with various ligands and aspect-ratio. 
LSPR of aqueous ellipsoid nanoparticles showed a linear correlation between peak position and the AR as follows:

$$
\lambda_{\max }=420+95 \mathrm{AR}^{26,27}
$$

Thus, the blue shift of the spectrum caused by the difference in aspect ratio is $<10 \mathrm{~nm}$, indicating that the blue shift might be caused by surface modification. The profile of the experimental spectra, as well as the full width at half maximum of longitudinal LSPR peak, was maintained constant, indicating the effective exchange of the CTAB bilayer without inducing aggregation. The ligand exchange results were also monitored by measuring their surface charge via the dynamic light scattering (DLS) method (Figure 1I). The zeta-potential of CTAB-stabilized AuNRs was positive surface charge due to the positive charge of the trimethylammonium group $[-\mathrm{N}$ $\left.\left(\mathrm{CH}_{3}\right)_{3}{ }^{+}\right]$of CTAB, which showed a decreasing value with decreasing size: $62.3 \mathrm{mV}$ for CTAB-NRI, $40.2 \mathrm{mV}$, for CTABNRII, and $33.9 \mathrm{mV}$ for CTAB-NRIII. Following the PSS and $\mathrm{Na}_{3} \mathrm{CT}$ treatment, the zeta potential of CTAB-stabilized AuNRs shifted to a negative value. In this process, PSS was tightly adsorbed to the surface of the CTAB bilayer, and the PSS-CTAB complex could be isolated by centrifugation, forming citrate-stabilized AuNRs. Consequently, the surface charge becomes negative, indicating a successful replacement process. Similarly, as the aspect ratio decreases, the negative

A
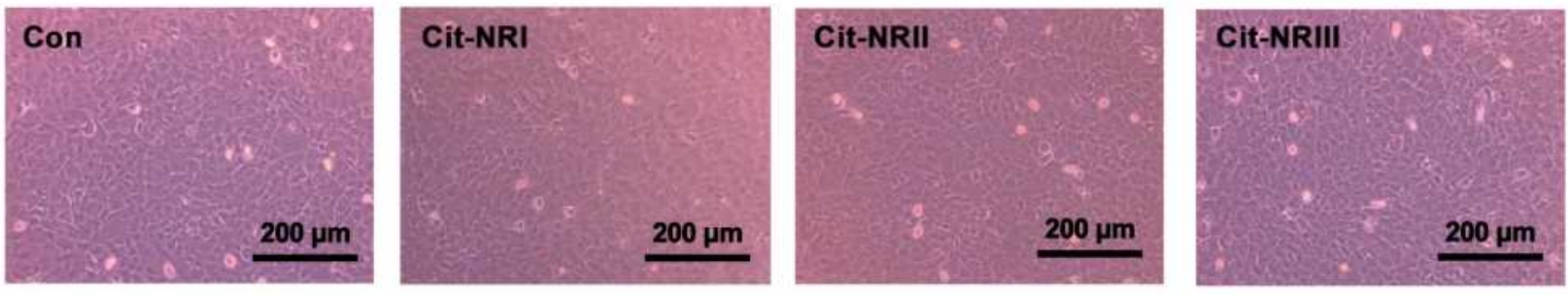

B
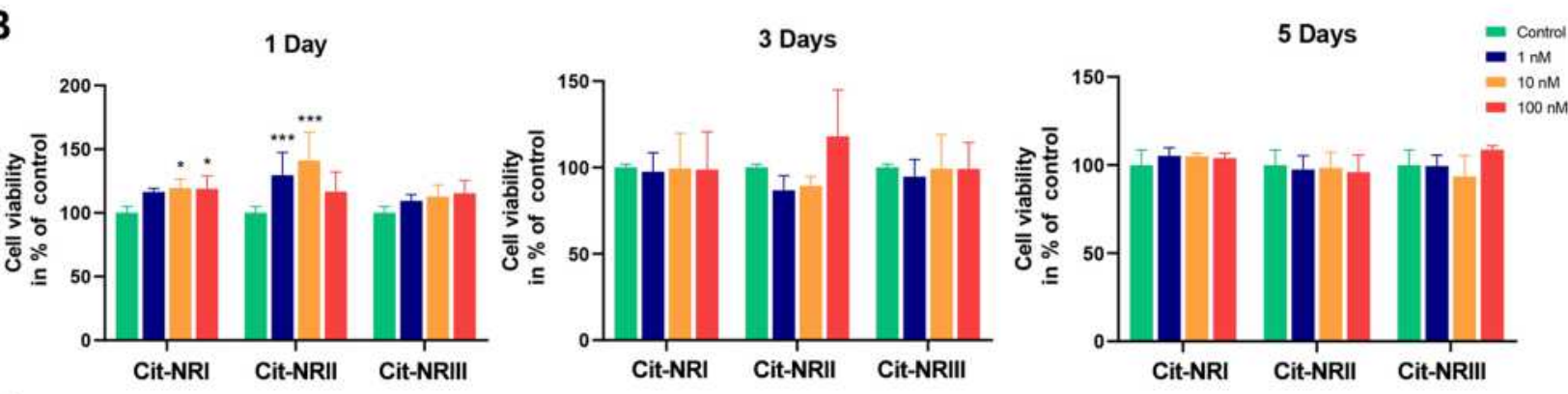

C
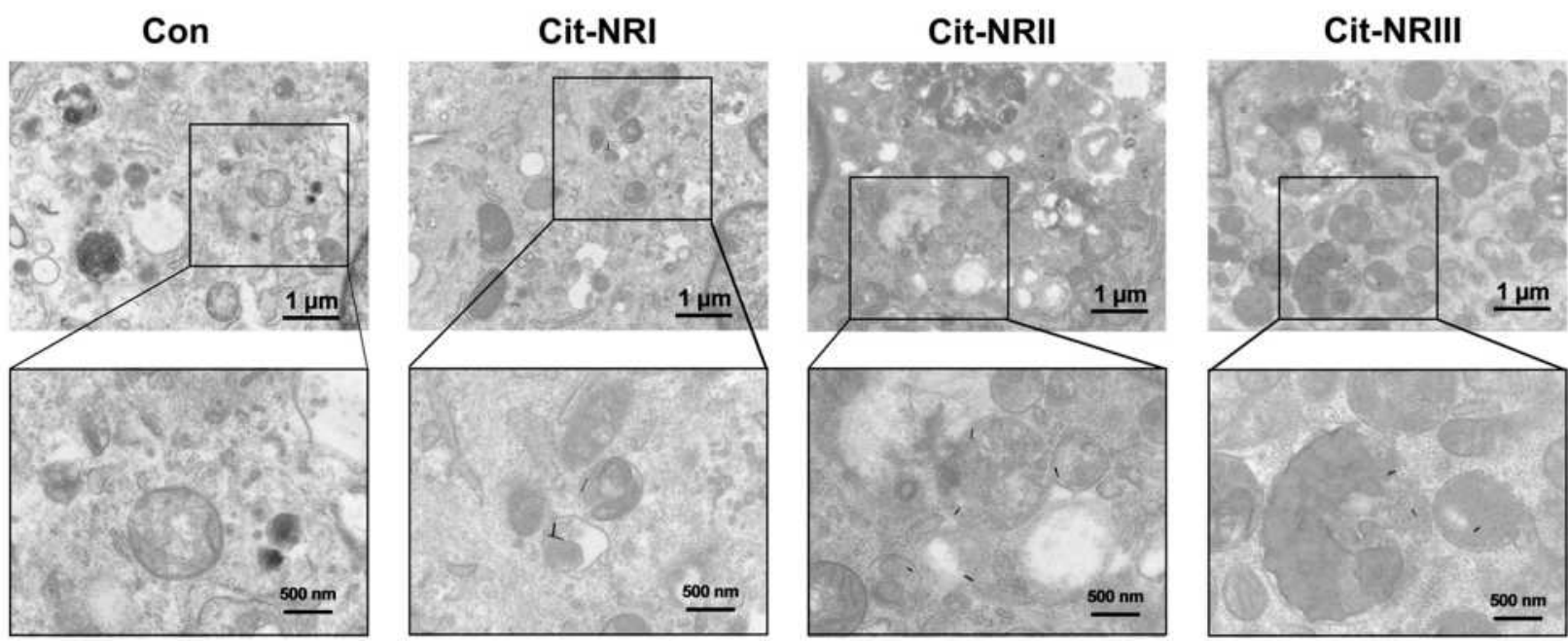

Figure 2 Biocompatibility and cellular uptake of citrate-stabilized AuNRs. (A) Photographs of MC3T3-EI cells treated with citrate-stabilized AuNRs with various aspectratio for $48 \mathrm{~h}$, respectively. (B) Cell viability of MC3T3-EI cells after incubated with citrate-stabilized AuNRs with various aspect-ratio for I, 3 and 5 days, respectively. (C) TEM images of MC3T3-EI cells treated with citrate-stabilized AuNRs (I00 nM) for I day. Asterisk indicates statistically significant differences between control and experience group $(* \mathrm{p}<0.05 ; * * * p 0.005)$. 
charge decreases. The CTAB- and citrate-stabilized AuNRs with various aspect-ratio were utilized to assess the biocompatibility and cellular internalization.

\section{Biocompatibility and Cellular Internalization of Citrate-Stabilized AuNRs}

The effects of CTAB-stabilized and citrate-stabilized AuNRs on biocompatibility and cellular internalization of MC3T3-E1 cells were investigated. As shown in Figure $\underline{\mathrm{S} 1 \mathrm{~A}-\mathrm{C}}$, the cell viability of MC3T3-E1 cells exceeds $95 \%$ in citrate-stabilized AuNRs groups after incubation for 1, 3 , and 5 days. Also, a slightly increased proliferation was observed after treatment with Cit-NRI and Cit-NRIII for 1 day. However, CTAB-stabilized AuNRs displayed some cytotoxicity. After 5 days of treatment, the viability decreased by $17.6 \%$ for CTAB-NRI and $38.9 \%$ for CTAB-NRII. These results were attributed to fact that the CTAB around the AuNR surface demonstrated potential toxicity to biological cells, which was in accordance with Wang's work. ${ }^{13}$ In addition, ARS staining assessed the potential effect on osteogenic differentiation of cells. As displayed in Figure S1D, more calcium nodules were observed in citrate-stabilized AuNRs groups compared to CTAB-stabilized AuNRs groups, which had an inhibitory effect on mineralization. These findings were further verified by quantified mineralized matrixes in the cells (Figure S1E). Based on the preliminary assessment of CTAB-stabilized and citrate-stabilized AuNRs on the biocompatibility and osteogenic differentiation of MC3T3-E1 cells, we found that citrate-stabilized AuNRs might be appropriately utilized as osteogenic bioactive material for bone regeneration. The comprehensive evaluation of their effects on osteogenic differentiation of MC3T3-E1 cells, rBMSCs, and PDLPs were further investigated.

Furthermore, the biocompatibilities of citrate-stabilized AuNRs with various aspect-ratio were tested by CCK8 assays. As shown in Figure 2A, after co-incubation with MC3T3-E1 cells for $48 \mathrm{~h}$ at the concentrations of 1,10 , and $100 \mathrm{nM}$, no obvious morphological changes were observed as compared to that in control group, which was in agreement with the results of rBMSCs (Figure S2A) and PDLPs (Figure S3A). Additionally, citrate-stabilized AuNRs did not exhibit any
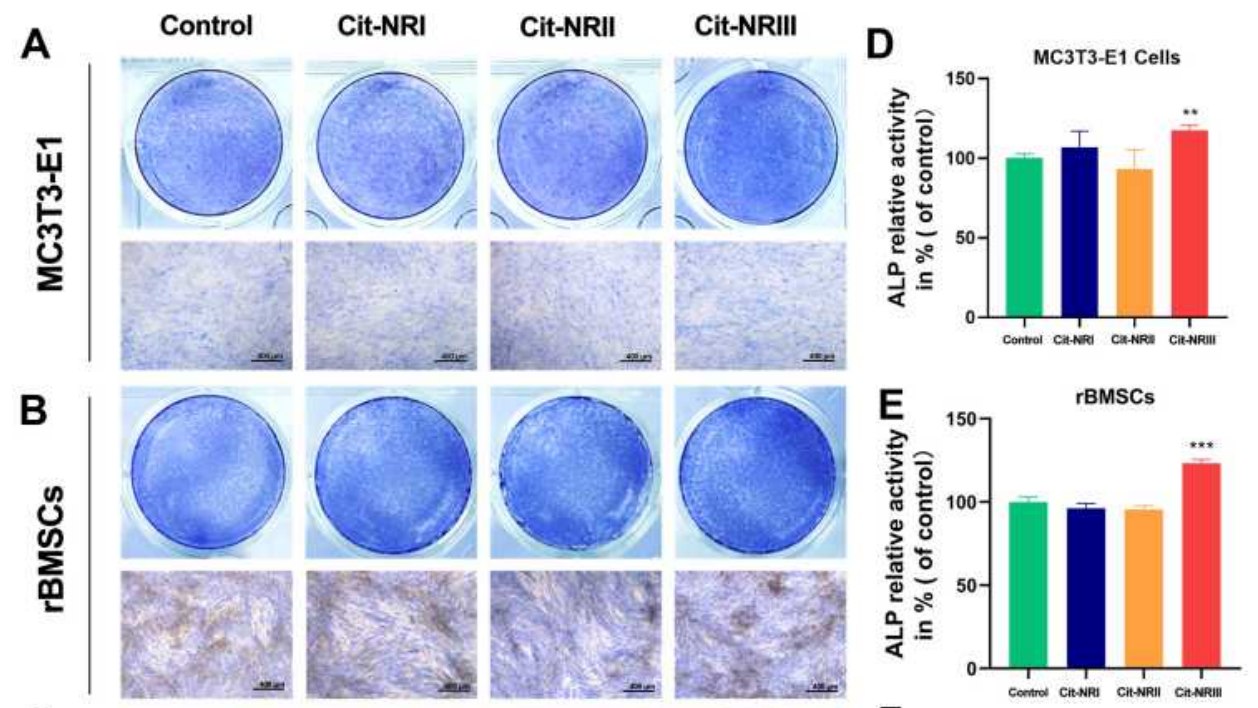

C
a
$\frac{0}{\alpha}$
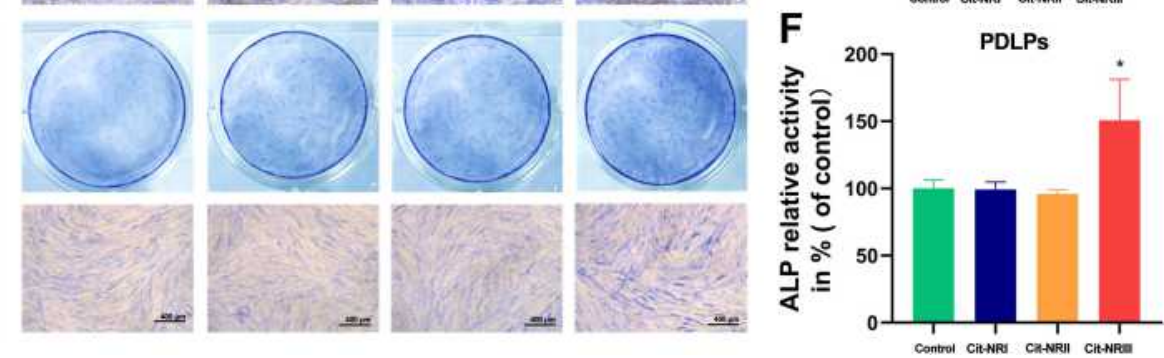

Figure 3 Effects of AuNRs on ALP activity. (A-C) ALP staining and (D-F) ALP activity level of MC3T3-EI cells, rBMSCs and PDLPs after treated with citrate-stabilized AuNRs with various aspect-ratio for 14 days, respectively. Asterisk indicates statistically significant differences between control and experience group $\left({ }^{*} \mathrm{p}<0.05 ; * * \mathrm{p}<0.0 \mathrm{I}\right.$; *** $\mathrm{p}<0.005)$. 
significant cytotoxicity to MC3T3-E1 cells (Figure 2B), rBMSCs (Figure S2B), and PDLPs (Figure S3B), indicating a viability of $>95 \%$ in all groups after incubation for 1,3 , and 5 days, respectively. These findings indicated that the citratestabilized AuNRs were non-toxic and exhibited adequate biocompatibility due to the citrate molecule around the AuNRs, which was in agreement with our previous work. ${ }^{28}$ It is known that the fate of gold nanomaterials after internalized by cells were significantly affected by their shape, size, and most importantly, surface functionalization. As for the conventional fate of citrate-stabilized gold nanomaterials after internalized by cells, which are internalized through membrane proteinsmediated endocytosis, localized in cytoplasm and finally excreted through exocytosis. The cellular uptake markedly affects the interactions between nanomaterials and biological cells, wherein thermodynamics plays a dominant role in the cellular internalization of nanomaterials. ${ }^{29}$ Spherical nanoparticles with size $<50 \mathrm{~nm}$ cluster to generate a driving force for cellular uptake, while the size $>50 \mathrm{~nm}$ is not required. ${ }^{30}$ To further investigate the cellular internalization of citrate-stabilized AuNRs with various aspect-ratio, TEM was performed and the data are shown in Figure 2C, where it could be seen that all the three types of AuNRs were taken up by cells and localized in intracellular vesicles, instead of other cellular organelles, such as mitochondria and cell nucleus after treatment with MC3T3-E1 cells for $24 \mathrm{~h}$. As expected, the AuNRs in vesicles were insulated as their size was $>50 \mathrm{~nm}$, which is consistent with the data from previous studies. ${ }^{31}$ Due to the anisotropic structure of AuNRs, the permeability in the cells need excess energy, which makes it difficult for the intracellular uptake compared to that of spherical nanoparticles. Besides, the citrate-stabilized AuNRs possessed extreme stability, which might be hard to degrade. Therefore, we concluded that citrate-stabilized AuNRs might be partly excreted from cells through exocytosis but not be degraded.

\section{Effects of Citrate-Stabilized AuNRs on ALP Activity and Mineralization}

ALP is an early bone marker (7-14 days) that represents the osteogenic activity of cells. ${ }^{32}$ To investigate the effects of citrate-stabilized AuNRs with various aspect-ratio on

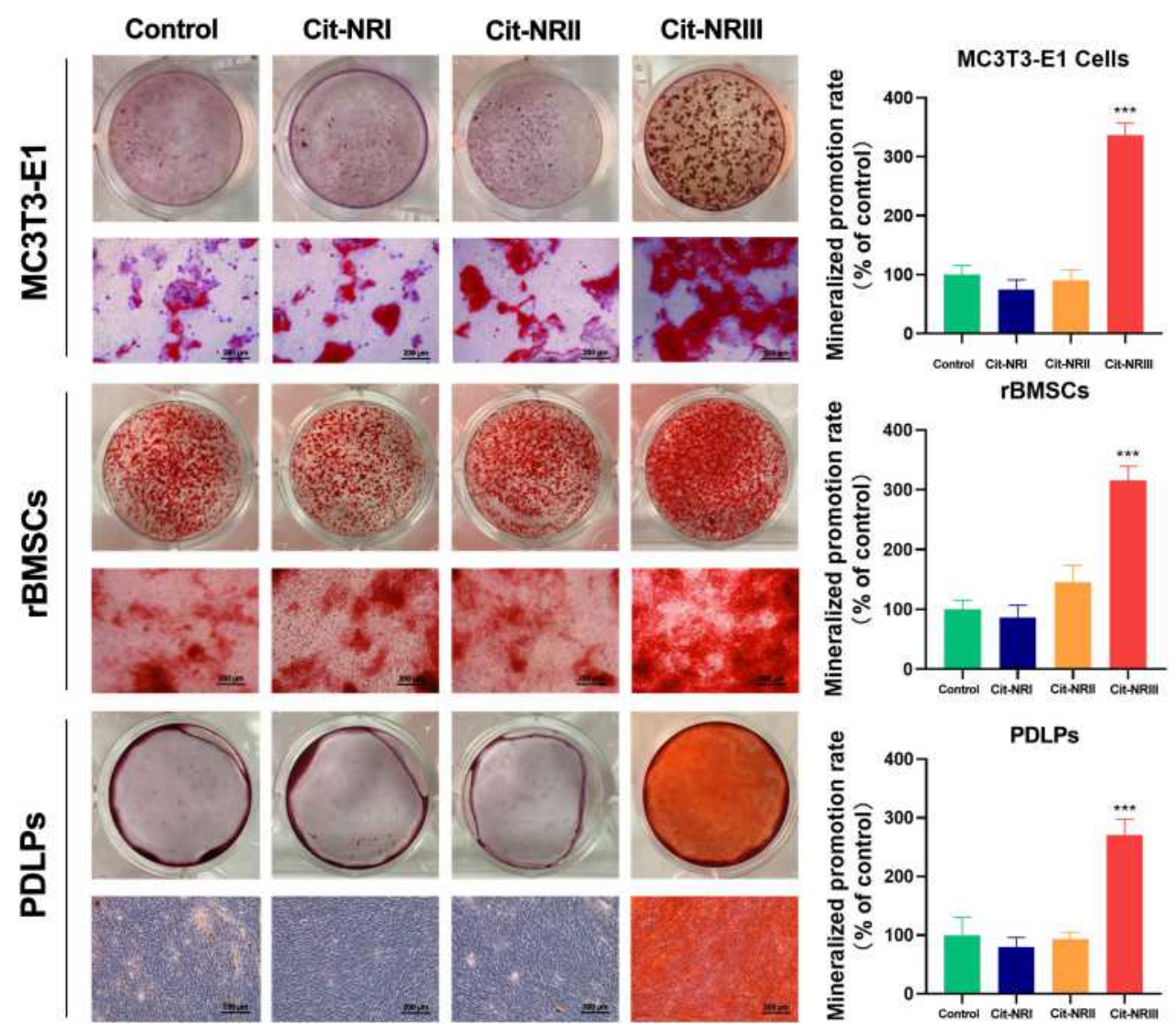

Figure 4 Effects of AuNRs on mineralization. Mineralized nodules stained with alizarin red S and calcium deposition assay of MC3T3-EI cells, rBMSCs and PDLPs after incubated with citrate-stabilized AuNRs with various aspect-ratio for 21 days, respectively. Asterisk indicates statistically significant differences between control and experience group $(* * * p<0.005)$. 
osteogenic differentiation of cells, the ALP staining of MC3T3-E1 cells (Figure 3A), rBMSCs (Figure 3B), and PDLPs (Figure 3C) was assessed after treatment with citrate-stabilized AuNRs for 14 days. As a result, the ALP expression in all three types of cells was enhanced in the CitNRIII group, while no significant difference was detected in the Cit-NRI and Cit-NRII groups. Moreover, the quantitative analysis of ALP activity levels after treatment was further detected, which revealed a similar trend, as shown in Figure 3D-F. Among the three types of citrate-stabilized AuNRs, Cit-NRIII had the strongest ability to increase the ALP activity level, indicating that the effects of citrate-stabilized AuNRs on the ALP activity levels in the cells were aspectratio-dependent. Furthermore, the formation of mineralized nodules is a critical phenotypic marker that reflects the final stage of the osteogenic differentiation of cells. ${ }^{33}$ The calcium depositions were evaluated by ARS staining for three types of cells cultured with citrate-stabilized AuNRs with various aspect-ratio in the osteogenic induction medium for 21 days, and the data are shown in Figure 4. However, no significant difference was detected among the Cit-NRI, Cit-NRII, and control groups, while calcium nodules were observed in the
Cit-NRIII group, indicating a robust effect on mineralization. Furthermore, the mineralized matrixes in the cells were further quantified by eluting and measuring the absorbance of ARS deposits at $562 \mathrm{~nm}$. The results confirmed that the effects of citrate-stabilized AuNRs on intracellular calcium nodules were also aspect-ratio-dependent, whereas Cit-NRIII exhibited stronger effects compared to Cit-NRI and CitNRII.

\section{Effects of Citrate-Stabilized AuNRs on Osteogenic Gene Expression by Activating the $\mathrm{Wnt} / \beta$-Catenin Signaling Pathway}

Several specific marker genes, such as $A L P$, Runt-related Transcription Factor 2 (Runx2), type I Collagen (COL1), and Osteopontin $(O P N)$, were inevitably expressed during the process of osteogenic differentiation. ${ }^{34,35}$ Among these osteogenic-related genes, $A L P$ is a hydrolase enzyme that supplies calcium nucleation sites with inorganic phosphate and Runx2, a key transcription factor; together, these are considered as early markers of osteogenic differentiation. ${ }^{36}$
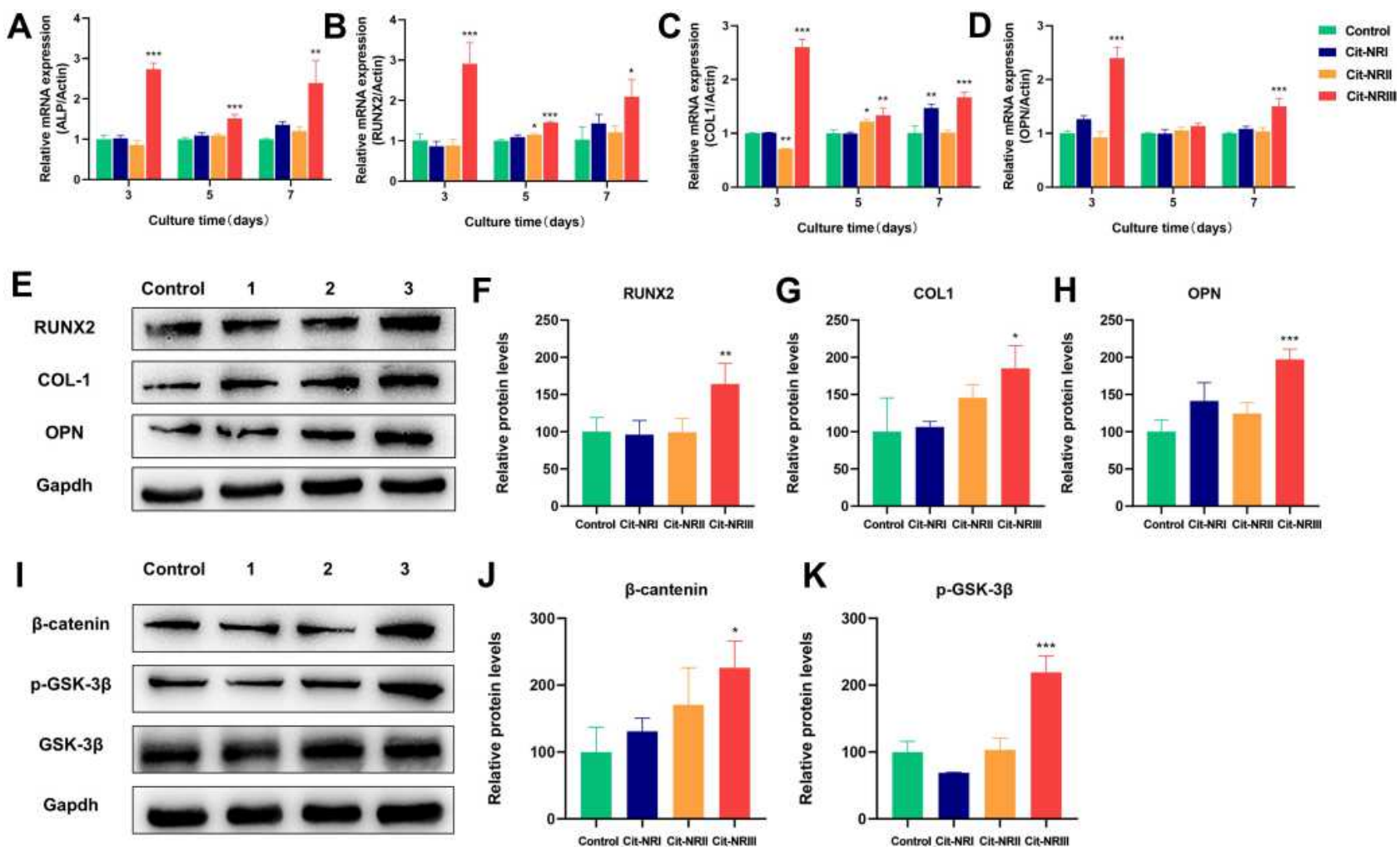

Figure 5 Effects of GNPs on osteogenic gene expression via Wnt//-catenin signal pathway. MC3T3-EI cells were treated with citrate-stabilized AuNRs with various aspectratio for 3, 5 and 7 days. (A-D) Real-time PCR analysis of ALP, Runx-2, COLI and OPN expressions. (E) Western blot analysis and (F-H) relative protein level of Runx-2, COLI and OPN expressions. (I) Western blot analysis and ( $\mathbf{J}$ and $\mathbf{K})$ relative protein level of $\beta$-catenin and $\mathrm{p}$-GSK-3 $\beta$ expressions. Asterisk indicates statistically significant differences between control and experience group $\left({ }^{*} \mathrm{p}<0.05 ;{ }^{* *} \mathrm{p}<0.01\right.$; $\left.{ }^{* * *} \mathrm{p}<0.005\right)$. 
COL1 is the main component responsible for forming the bone extracellular matrix, and OPN is a key marker that regulates osteoblast differentiation, the formation of mineralized nodules, and bone-building. ${ }^{37}$ We further investigated these osteogenic-related genes expression level by real-time polymerase chain reaction (PCR). As shown in Figure 5A-D, after co-incubation with citrate-stabilized AuNRs for 3, 5, and 7 days, the mRNA expression level of these osteogenic genes was significantly upregulated by one- to threefold under Cit-NRIII treatment and was associated with progressive osteogenic differentiation of MC3T3-E1 cells, while no significant differences were detected in Cit-NRI- and CitNRII-treated groups. These results were similar to those of the ALP activity and mineralization. WB revealed that the expression level of these osteogenic-related genes and proteins, COL-1, Runx-2, and OPN, was upregulated after treatment with Cit-NRIII (Figure 5E-H). These findings indicated that Cit-NRIII enhances the osteogenic differentiation of MC3T3-E1 cells compared to that of Cit-NRI and CitNRII in vitro. Furthermore, Runx2 is considered as a key downstream gene that is involved in the Wnt/ $\beta$-catenin signaling pathway during the osteogenic differentiation of cells. ${ }^{38}$ As the upregulated of Runx2 is induced by CitNRIII, we speculated that the Wnt/ $\beta$-catenin signaling pathway might play an important role in Cit-NRIII-induced osteogenic differentiation in MC3T3-E1 cells. Wnt/ $\beta$-catenin signaling pathway regulates the osteogenic differentiation of cells, wherein the accumulation of $\beta$-catenin in the cytoplasm determines its activation. ${ }^{39}$ Moreover, $\beta$-catenin is phosphorylated by GSK-3 $\beta$, a serine/threonine-protein kinase, resulting in the inactivation and degradation in cytoplasm. Upon Wnt activation, GSK-3 $\beta$ was inhibited via phosphorylation of Ser9, causing the accumulation of $\beta$-catenin in the cytoplasm, thus improving the expression of Runx $2 .^{40}$ Therefore, potential molecular mechanisms were investigated by examining the expression of key markers, such as $\beta$-catenin and $\mathrm{p}$-GSK-3 $\beta$, which might be involved in the relevant signaling pathways during the osteogenic differentiation of MC3T3-E1 cells. ${ }^{41,42}$ As expected, WB analysis demonstrated that the level of $\beta$-catenin and $p$-GSK-3 $\beta$ was increased in Cit-NRIII-treated cells (Figure 5I-K), indicating that the $\mathrm{Wnt} / \beta$-catenin signaling pathway might play a major role in citrate-stabilized AuNRs-induced osteogenic differentiation in MC3T3-E1 cells.

\section{Mechanisms of Osteogenic Effect Induced by Citrate-Stabilized AuNRs}

The mechanisms of Cit-NRIII-induced osteogenic differentiation of MC3T3-E1 cells were further validated by specific Wnt/ $\beta$-catenin signaling inhibitor, KYA1797K,
A

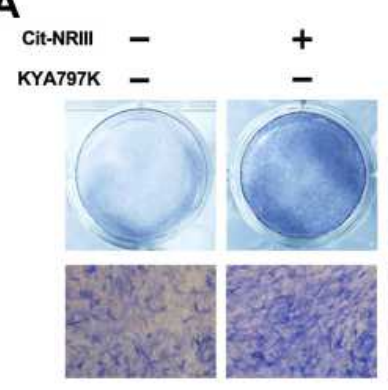

E

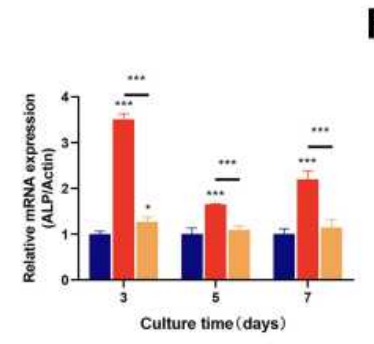

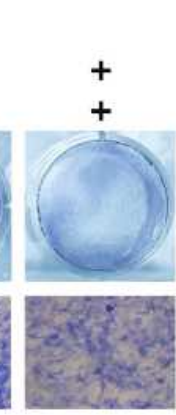

$\mathbf{F}$
B

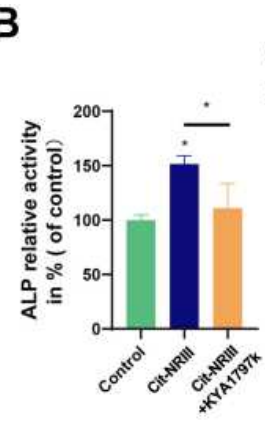

G

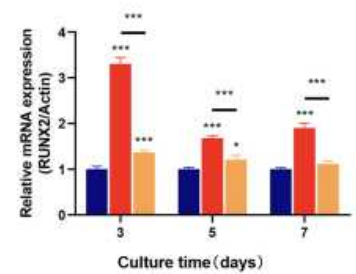

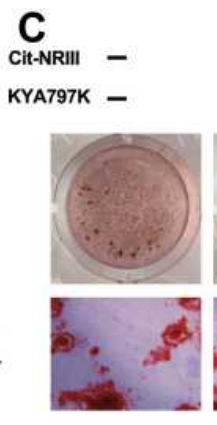

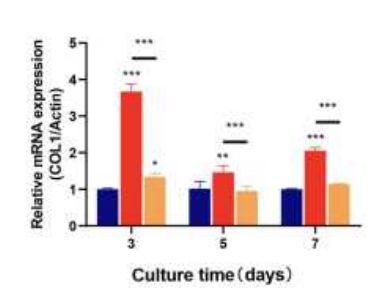

D
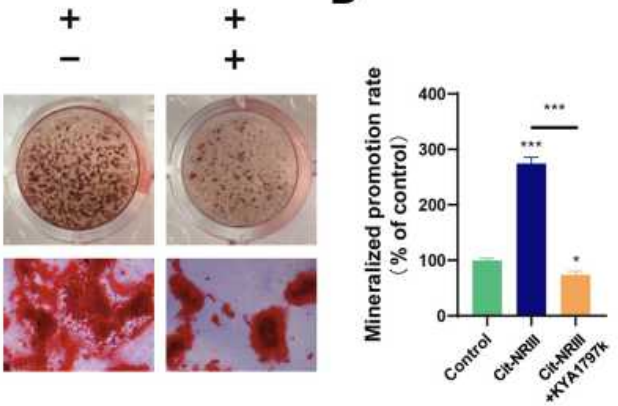

H

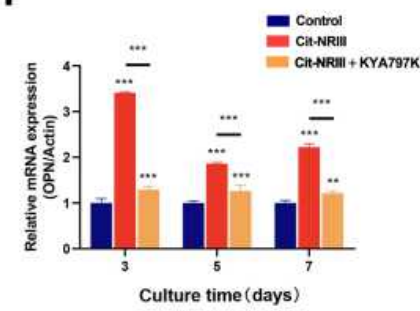

Figure 6 Effects of Wnt/ $\beta$-catenin inhibitor KYAI797K on Cit-NRIII-induced osteogenic differentiation. (A) ALP staining and (B) ALP activity level of MC3T3-EI cells after treated with Cit-NRIII alone or combine with KYAI797K for I4 days, respectively. (C) Mineralized nodules stained with alizarin red S and (D) Calcium deposition assay of MC3T3-EI cells after treated with Cit-NRIII alone or combine with KYAI797K for 21 days, respectively. (E-H) Real-time PCR analysis of ALP, Runx2, COLI and OPN, mRNA expression of MC3T3-EI cells after treated with Cit-NRIII alone or combine with KYAI797K for 3, 5 and 7 days, respectively. Asterisk indicates statistically significant differences between control and experience group $\left({ }^{*} \mathrm{p}<0.05 ;{ }^{* *} \mathrm{p}<0.01\right.$; $\left.{ }^{* * *} \mathrm{p}<0.005\right)$. 
which could markedly decrease the activity of $\beta$-catenin involved in $\mathrm{Wnt} / \beta$-catenin signaling pathway. ${ }^{43}$ As a low molecular weight compound, KYA1797K was firstly studied its effects on surface of citrate-stabilized AuNRs. UV-Vis absorption spectra of citrate-stabilized AuNRs before and after co-incubated with KYA1797K for 24, 48 and 72 hours were measured. As shown in Figure S4, the absorption peak also existed but showed red shift of $9 \mathrm{~nm}$ when incubated with KYA1797K, which might be due to the absorption of KYA1797K on surface of nanorods. These results indicated that the citrate-stabilized AuNRs still keep stability and their surface are not be obviously disturbed. As shown in Figure 6A and B, the enhanced ALP activity induced by Cit-NRIII was significantly blocked with KYA1797K. Consistent with this, ARS staining (Figure 6C) and quantified mineralized matrixes inside cells (Figure 6D) showed that the mineralized-nodule formation after treatment with Cit-NRIII combined with
KYA1797K was lower than that with Cit-NRIII alone. Also, the qRT-PCR analysis revealed that Cit-NRIII combined with KYA1797K treatment resulted in a downregulation of the expression of osteogenic-related genes, ALP, RUNX2, COL1, and OPN combined with the Cit-NRIII alone group (Figure 6E-H). In addition, Western blots (Figure 7A) demonstrated that the effects of Cit-NRIII on osteogenic-related protein expression (RUNX2, COL1, and OPN) were reversed by KYA1797K as shown in Figure 7B-D. Surprisingly, $\beta$ catenin and p-GSK-3 $\beta$, the critical proteins in $W n t / \beta$ catenin signaling pathway, were also examined by WB (Figure 7E-G). Consequently, the expression of these proteins was enhanced significantly by treatment with Cit-NRIII and immediately blocked by KYA1797K treatment. The inhibitor KYA1797K reversed the enhanced osteogenic differentiation induced by CitNRIII, indicating that Cit-NRIII-induced acceleration of osteogenic differentiation of MC3T3-E1 cells was

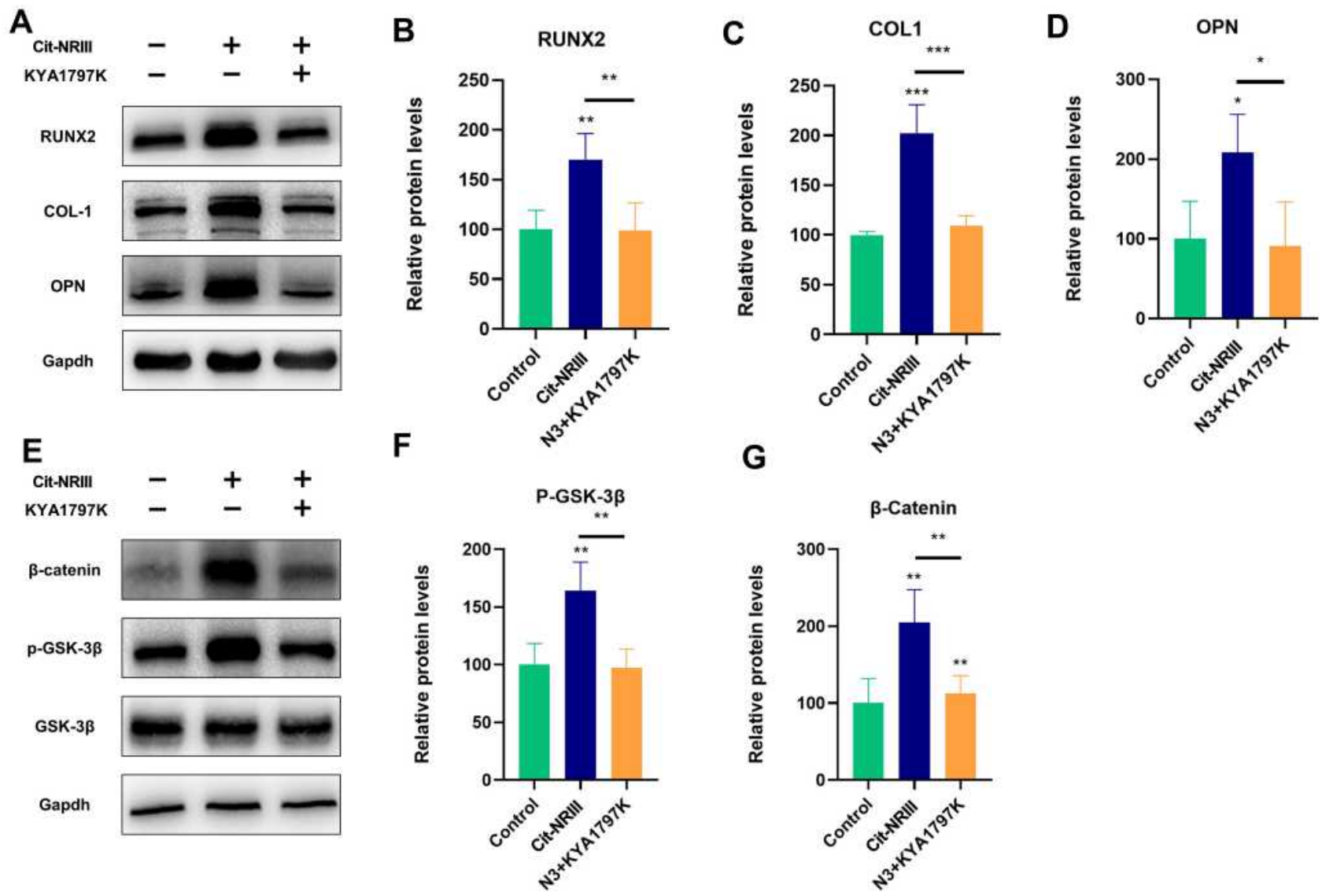

Figure 7 Effects of Wnt/ $\beta$-catenin inhibitor KYAI797K on Cit-NRIII-induced osteogenic differentiation. MC3T3-EI cells were treated with Cit-NRIII alone or combine with KYAI797K for 3 days. (A) Western blot analysis and (B-D) relative protein level of Runx-2, COLI and OPN expressions. (E) Western blot analysis and (F and $\mathbf{G})$ relative protein level of $\beta$-catenin and p-GSK-3 $\beta$ expression. Asterisk indicates statistically significant differences between control and experience group $\left({ }^{*} \mathrm{p}<0.05 ; * * \mathrm{p}<0.0 \mathrm{I}\right.$; $* * * \mathrm{p}<$ 0.005). 
related to the activation of the $\mathrm{Wnt} / \beta$-catenin signaling pathway. These results indicated that Cit-NRIII-induced osteogenic differentiation might be regulated via $\mathrm{Wnt} / \beta$ catenin signaling pathway.

\section{Conclusions}

In summary, citrate-stabilized AuNRs with various aspectratios showed excellent biocompatibility and their effects on osteogenic differentiation of Multiple Cells were aspect-ratio-dependent, where Cit-NRIII demonstrated enhanced ALP activity, mineralized-nodule formation and expression of osteogenic-related genes and proteins. The mechanisms underlying citrate-stabilized AuNRs-induced osteogenic differentiation of MC3T3-E1 cells revealed that $\mathrm{Wnt} / \beta$-catenin signaling pathway might provide a potential explanation for the citrate-stabilized AuNRs-mediated osteogenic differentiation. These findings could elucidate the contribution of citrate-stabilized AuNRs in modulating osteogenic differentiation of cells, which were also utilized as potential osteogenic candidates in the field of bone tissue engineering and regenerative medicine.

\section{Abbreviations}

AuNRs, gold nanorods; CTAB, cetyltrimethylammonium bromide; TEM, transmission electron microscopy; DLS, dynamic light scattering; MC3T3-E1, pre-osteoblastic cell line; rBMSCs, rat bone marrow mesenchymal stem cells; PDLPs, human periodontal ligament progenitor cells; PCR, real-time polymerase chain reaction; Runx2, Runt-related Transcription Factor 2; COL1, type I Collagen; OPN, Osteopontin.

\section{Funding}

This work was supported by National Natural Science Foundation of China (81802135, 81972124, 81730067, and $81420108021)$, the Natural Science Foundation of Jiangsu Province (Nos. BK20200121 and $\backslash$, 20170123), Nanjing University Innovation Program for $\mathrm{PhD}$ candidate (CXYJ2162), Key project in Medical science and Technology Development of Nanjing (ZKS18020), National Key Research and Development Project (Grant No. 2018YFF0301100) and 789 Outstanding Talent Program of SAHNMU.

\section{Disclosure}

The authors report no conflicts of interest for this work.

\section{References}

1. Gu J, Guo M, Huang C, et al. Titanium dioxide nanoparticle affects motor behavior, neurodevelopment and axonal growth in zebrafish (Danio rerio) larvae. Sci Total Environ. 2021;754:142315. doi:10.1016/j.scitotenv.2020.142315

2. Yu P, Zheng L, Wang $P$, et al. Development of a novel polysaccharide-based iron oxide nanoparticle to prevent iron accumulation-related osteoporosis by scavenging reactive oxygen species - ScienceDirect. Int J Biol Macromol. 2020;165:1634-1645. doi:10.1016/j.ijbiomac.2020.10.016

3. Zhang Y, Wang P, Wang Y, et al. Gold nanoparticles promote the bone regeneration of periodontal ligament stem cell sheets through activation of autophagy. Int $J$ Nanomedicine. 2021;16:61-73. doi:10.2147/IJN.S282246

4. Hashemzadeh H, Allahverdi A, Ghorbani M, et al. Gold nanowires/ fibrin nanostructure as microfluidics platforms for enhancing stem cell differentiation: Bio-AFM Study. Micromachines. 2020;11(1):50. doi: $10.3390 / \mathrm{mi} 11010050$

5. Wang P, Ma S, Ning G, et al. Entry-prohibited effect of $\mathrm{kHz}$ pulsed magnetic field upon interaction between SPIO nanoparticles and mesenchymal stem cells. IEEE Trans Biomed Eng. 2019;99:1.

6. Hashemzadeh H, Allahverdi A, Sedghi $M$, et al. PDMS nano-modified scaffolds for improvement of stem cells proliferation and differentiation in microfluidic platform. Nanomaterials. 2020;10 (4):668. doi:10.3390/nano10040668

7. Letchumanan I, Arshad MK, Balakrishnan SR, et al. Gold-nanorod enhances dielectric voltammetry detection of c-reactive protein: a predictive strategy for cardiac failure. Biosens Bioelectron. 2019;130:40-47. doi:10.1016/j.bios.2019.01.042

8. Zheng ZK, Tachikawa T, Majima T. Single-particle study of pt-modified au nanorods for plasmon-enhanced hydrogen generation in visible to near-infrared region. $J$ Am Chem Soc. 2014;136 (19):6870-6873. doi:10.1021/ja502704n

9. Huang XH, EI-Sayed IH, Qian W, et al. Cancer cell imaging and photothermal therapy in the near-infrared region by using gold nanorods. J Am Chem Soc. 2006;128(6):2115-2120. doi:10.1021/ ja057254a

10. Chen YS, Zhao Y, Yoon SJ, et al. Miniature gold nanorods for photoacoustic molecular imaging in the second near-infrared optical window. Nat Nanotechnol. 2019;14(5):465-472. doi:10.1038/s41565019-0392-3

11. Yuan D, Yan H, Liu J, et al. A fast and colorimetric sensor array for the discrimination of ribonucleotides in human urine samples by gold nanorods. Chin Chem Lett. 2020;31(2):455-458. doi:10.1016/j. cclet.2019.07.067

12. Nikoobakht B, El-Sayed MA. Preparation and growth mechanism of gold nanorods (NRs) using seed-mediated growth method. Chem Mater. 2003;15(10):1957-1962. doi:10.1021/cm0207321

13. Wang LM, Jiang XM, Ji Y, et al. Surface chemistry of gold nanorods: origin of cell membrane damage and cytotoxicity. Nanoscale. 2013;5 (18):8384-8391. doi:10.1039/c3nr01626a

14. Gole A, Murphy CJ. Polyelectrolyte-coated gold nanorods: synthesis, characterization and immobilization. Chem Mater. 2005;17 (6):325-1330. doi:10.1021/cm048297d

15. Gorelikov I, Matsuura N. Single-step coating of mesoporous silica on cetyltrimethyl ammonium bromide-capped nanoparticles. Nano Lett. 2008;8(1):369-373. doi:10.1021/n10727415

16. Bai YY, Zheng SY, Zhang LM, et al. Non-invasively evaluating therapeutic response of nanorod-mediated photothermal therapy on tumor angiogenesis. J Biomed Nanotechnol. 2014;10(11):3351-3360. doi:10.1166/jbn.2014.1994 
17. Vieira S, Vial S, Maia FR, et al. Gellan gum-coated gold nanorods: an intracellular nanosystem for bone tissue engineering. RSC $A d v$. 2015;95(95):77996-78005. doi:10.1039/C5RA13556G

18. Carbo-Argibay E, Rodriguez-Gonzalez B, Gomez-Grana S, et al. The crystalline structure of gold nanorods revisited: evidence for higher-index lateral facets. Angew Chem Int Ed. 2010;49 (49):9397-9400. doi:10.1002/anie.201004910

19. Mehtala JG, Zemlyanov DY, Max JP, et al. Citrate-stabilized gold nanorods. Langmuir. 2014;30(46):13727-13730. doi:10.1021/ la5029542

20. Li JC, Li J, Zhang J, et al. Gold nanoparticle size and shape influence on osteogenesis of mesenchymal stem cells. Nanoscale. 2016;8 (15):7992-8007. doi:10.1039/C5NR08808A

21. Zhang BJ, Xia YS. Etching of gold nanorods: the effects of diameter on analytical performances. Chin Chem Lett. 2019;30(9):1663-1666. doi:10.1016/j.cclet.2019.06.038

22. Nikoobakht B, El-Sayed MA. Evidence for bilayer assembly of cationic surfactants on the surface of gold nanorods. Langmuir. 2001;17(20):6368-6374. doi:10.1021/la010530o

23. Zhang KZ, Bai TT, Wang M, et al. Refractive index sensitivity and SERS ability of citrate versus CTAB-stabilized gold nanorods: a Comparative Study. Nanosci Nanotechnol Lett. 2018;10 (1):119-126. doi:10.1166/nnl.2018.2584

24. Li WH, Guo ZR, Tai QY, et al. Rapid and fine tailoring longitudinal surface plasmon resonances of gold nanorods by end-selective oxidation. Chin Chem Lett. 2020;31(9):2447-2451. doi:10.1016/j. cclet.2020.05.019

25. Wang P, Sun WJ, Wang Q, et al. Iodine-labeled au nanorods with high radiochemical stability for imaging-guided radiotherapy and photothermal therapy. ACS Appl Nano Mater. 2019;2(3):1374-1381. doi:10.1021/acsanm.8b02229

26. Huang Y, Xia K, He N, et al. Size-tunable synthesis of gold nanorods using pyrogallol as a reducing agent. Sci China Chem. 2015;58 (11):1759-1765. doi:10.1007/s11426-015-5437-3

27. Link S, El-Sayed MA. Simulation of the optical absorption spectra of gold nanorods as a function of their aspect ratio and the effect of the medium dielectric constant. J Phys Chem B. 2005;109 (20):10531-10532. doi:10.1021/jp058091f

28. Zhang Y, Wang $\mathrm{P}$, Mao $\mathrm{H}$, et al. PEGylated gold nanoparticles promote osteogenic differentiation in in vitro and in vivo systems. Mater Des. 2020;197:109231. doi:10.1016/j.matdes.2020.109231

29. Alkilany AM, Murphy CJ. Toxicity and cellular uptake of gold nanoparticles: what we have learned so far? J Nanopart Res. 2010;12(7):2313-2333. doi:10.1007/s11051-010-9911-8

30. Zhang YH, Kong N, Zhang YC, et al. Size-dependent effects of gold nanoparticles on osteogenic differentiation of human periodontal ligament progenitor cells. Theranostics. 2017;7(5):1214-1224. doi: $10.7150 /$ thno. 17252

31. Chithrani BD, Chan WCW. Elucidating the mechanism of cellular uptake and removal of protein-coated gold nanoparticles of different sizes and shapes. Nano Lett. 2007;7(6):1542-1550. doi:10.1021/n1070363y
32. Wang QW, Chen B, Cao M, et al. Response of MAPK pathway to iron oxide nanoparticles in vitro treatment promotes osteogenic differentiation of hBMSCs. Biomaterials. 2016;86:11-20. doi:10.1016/j. biomaterials.2016.02.004

33. Zhang KY, Lin S, Feng Q, et al. Nanocomposite hydrogels stabilized by self-assembled multivalent bisphosphonate-magnesium nanoparticles mediate sustained release of magnesium ion and promote in-situ bone regeneration. Acta Biomater. 2017;64:389-400. doi:10.1016/j. actbio.2017.09.039

34. Pajarinen J, Lin T, Gibon E, et al. Mesenchymal stem cell-macrophage crosstalk and bone healing. Biomaterials. 2019;196:80-89. doi:10.1016/j.biomaterials.2017.12.025

35. Wang QW, Chen B, Ma F, et al. Magnetic iron oxide nanoparticles accelerate osteogenic differentiation of mesenchymal stem cells via modulation of long noncoding RNA INZEB2. Nano Res. 2017;10 (2):626-642. doi:10.1007/s12274-016-1322-4

36. Logan N, Brett P. The control of mesenchymal stromal cell osteogenic differentiation through modified surfaces. Stem Cells Int. 2013;2013:1-10. doi:10.1155/2013/361637

37. Feng B, Weng J, Yang BC, et al. Characterization of titanium surfaces with calcium and phosphate and osteoblast adhesion. Biomaterials. 2004;25(17):3421-3428. doi:10.1016/j. biomaterials.2003.10.044

38. Yu AXD, Xu ML, Yao P, et al. Corylin, a flavonoid derived from psoralea fructus, induces osteoblastic differentiation via estrogen and Wnt/beta-catenin signaling pathways. FASEB J. 2020;34 (3):4311-4328. doi:10.1096/fj.201902319RRR

39. Feng YM, Wan P, Yin L, et al. The inhibition of MicroRNA-139-5p promoted osteoporosis of bone marrow-derived mesenchymal stem ceils by targeting wnt/beta-catenin signaling pathway by NOTCH1. J Microbiol Biotechnol. 2020;30(3):448-458. doi:10.4014/ jmb.1908.08036

40. Wang T, Sun CH, Zhong HB, et al. N-(3-methoxybenzyl)(9Z,12Z,15Z)-octadecatrienamide promotes bone formation via the canonical Wnt/beta-catenin signaling pathway. Phytother Res. 2019;33(4):1074-1083. doi:10.1002/ptr.6301

41. Xiong LB, Liu Y, Zhu F, et al. Acetyl-11-keto-beta-boswellic acid attenuates titanium particle-induced osteogenic inhibition via activation of the GSK-3 beta/beta-catenin signaling pathway. Theranostics. 2019;9(24):7140-7155. doi:10.7150/thno.35988

42. Zhao SJ, Kong FQ, Jie J, et al. Macrophage MSR1 promotes BMSC osteogenic differentiation and M2-like polarization by activating PI3K/AKT/GSK3 beta/beta-catenin pathway. Theranostics. 2020;10 (1):17-35. doi:10.7150/thno.36930

43. Wang YQ, Wang N, Luo Y, et al. Ganoderal A effectively induces osteogenic differentiation of human amniotic mesenchymal stem cells via cross-talk between Wnt/beta-catenin and BMP/SMAD signaling pathways. Biomed Pharmacother. 2020;123:109807. doi:10.1016/j.biopha.2019.109807
International Journal of Nanomedicine

\section{Publish your work in this journal}

The International Journal of Nanomedicine is an international, peerreviewed journal focusing on the application of nanotechnology in diagnostics, therapeutics, and drug delivery systems throughout the biomedical field. This journal is indexed on PubMed Central, MedLine, CAS, SciSearch ${ }^{\mathbb{R}}$, Current Contents ${ }^{\mathbb{B}} /$ Clinical Medicine,
Journal Citation Reports/Science Edition, EMBase, Scopus and the Elsevier Bibliographic databases. The manuscript management system is completely online and includes a very quick and fair peer-review system, which is all easy to use. Visit http://www.dovepress.com/ testimonials.php to read real quotes from published authors. 